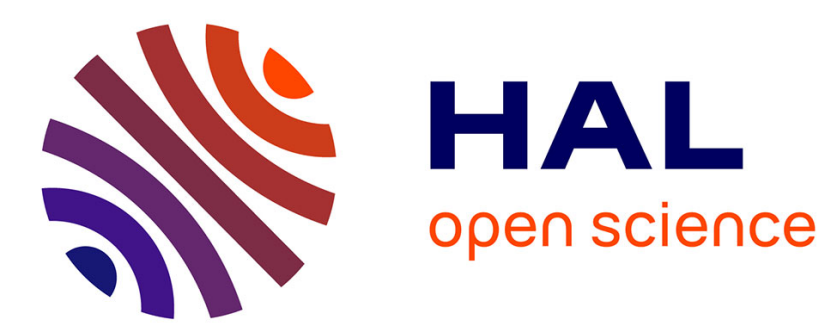

\title{
Equilibrium and stability of non-linearly elastic bodies with cavities containing fluid
}

\author{
V. A. Yeremeyev, L. M. Zubov
}

\section{To cite this version:}

V. A. Yeremeyev, L. M. Zubov. Equilibrium and stability of non-linearly elastic bodies with cavities containing fluid. Journal of Applied Mathematics and Mechanics, 1987, 51 (3), pp.353-356. hal00838369

\section{HAL Id: hal-00838369 \\ https://hal.science/hal-00838369}

Submitted on 25 Jun 2013

HAL is a multi-disciplinary open access archive for the deposit and dissemination of scientific research documents, whether they are published or not. The documents may come from teaching and research institutions in France or abroad, or from public or private research centers.
L'archive ouverte pluridisciplinaire HAL, est destinée au dépôt et à la diffusion de documents scientifiques de niveau recherche, publiés ou non, émanant des établissements d'enseignement et de recherche français ou étrangers, des laboratoires publics ou privés. 


\title{
EQUILIBRIUM AND STABILITY OF NON-LINEARLY ELASTIC BODIES WITH CAVITIES CONTAINING FLUID*
}

\author{
V.A. YEREMEYEV and L.M. ZUBOV
}

\begin{abstract}
Boundary conditions are formulated on the surface of a cavity filled with a compressible fluid or gas for the equilibrium problem of an elastic body experiencing large deformations. A formulation is given of the stability problem for the equilibrium of a non-linearly elastic body with fluid inclusions. The stability problem is solved for a thick-walled closed spherical shell filled with gas and loaded by external pressure.
\end{abstract}

1. We consider an elastic body occupying a volume $v$ in the reference configuration. Let the boundary of the domain $v$ consist of $m+1$ closed surfaces $\sigma, \sigma_{1}, \ldots, \sigma_{m}$, where $\sigma_{k}(k=1$, $2, \ldots m)$ are surfaces of simply-connected cavities, and $\sigma=\sigma^{\prime} \cup \sigma^{\prime \prime}$ is the outer boundary enclosing the body with the cavities. Each cavity is filled entirely with a compressible barotropic homogeneous liquid or gas. The body is deformed under the action of external forces distributed over parts of the surface $\sigma^{\prime}$. Displacements are given on the surface $\sigma^{*}$. We neglect the action of the mass forces. The pressure of the liquid is constant in each of *Prik1.Matem.Mekhan.,51,3,453-457,1987 
the cavities in the equilibrium state and the equilibrium equations for an elastic body are satisfied in the domain $v / 1 /$

$$
\nabla^{\circ} \cdot \mathbf{D}=0, \mathbf{D}=\partial W / \partial \mathbf{C}, \mathbf{C}=\nabla^{\circ} \mathbf{R}
$$

Here $\mathbf{R}$ is the radius-vector of points of the body in the deformed configuration, $\Gamma^{\circ} i s$ the nabla operator in the reference configuration, $\mathbf{C}$ is the site gradient, $W$ is the specific strain potential energy, and $\mathbf{D}$ is the Piola stress tensor. The boundary conditions on the boundary of the volume $v$ have the form

$$
\left.\mathbf{n} \cdot \mathbf{D}\right|_{\sigma^{\prime}}=\mathbf{t}_{0},\left.\quad \mathbf{R}\right|_{\sigma^{\prime \prime}}=\mathbf{R}_{0},\left.\quad \mathbf{n} \cdot \mathbf{D}\right|_{\sigma_{k}}=\left.p_{k} J \mathbf{n} \cdot \mathbf{C}^{-T}\right|_{\sigma_{k}}, \quad J=\operatorname{det} \mathbf{C}
$$

Here $t_{0}$ is the external load intensity per unit area in the reference configuration, $\mathbf{R}_{0}$ is a function given on $\sigma^{\prime \prime}, \mathbf{n}$ is the unit normal vector external to the volume $v$, and $p_{k}$ is the pressure in the liquid in the $k$-th cavity. Since $p_{k}$ are unknown in advance, the equations of

$$
p_{k}=\psi_{k}\left(\rho_{k}\right), \quad \rho_{k}=M_{k} / V_{k}
$$

state of the liquid must be used to obtain the closure of the set of boundary conditions, where $\rho_{k}$ is the liquid density in the $k$-th cavity, $M_{k}$ is its mass, and $V_{k}$ its volume in the deformed state. The volume $V_{k}$ is expressed in terms of the displacement of the cavity boundary by means of the formula

$$
V_{k}=-\frac{1}{3} \iint_{\Sigma_{k}} \mathbf{N} \cdot \mathbf{R} d \Sigma=-\frac{1}{3} \iint_{\sigma_{k}} J \mathbf{R} \cdot \mathbf{C}^{-1} \cdot \mathbf{n} d \boldsymbol{\sigma}
$$

(N is the normal to the surface $\Sigma_{k}$ bounding the cavity in the deformed state). Relationships result from (1.3) and (1.4) that close the system of boundary conditions on the elastic body boundary

$$
p_{k}=\psi_{k}\left[-3 M_{k}\left(\int_{\sigma_{k}} J \mathbf{R} \cdot \mathbf{C}^{-1} \cdot \mathbf{n} d \sigma\right)^{-1}\right]
$$

2. The stability of the solutions of problem (1.1), (1.2) and (1.5) can be investigated for conservative external forces by a static method consisting of studying the bifurcation of equilibrium. The linearized equilibrium equations and linearized boundary conditions on $\sigma$ have the form $/ 1 /$

$$
\nabla^{\circ} \cdot \mathbf{D}^{*}=0,\left.\quad \mathbf{n} \cdot \mathbf{D}^{*}\right|_{\sigma^{*}}=\mathbf{t}_{\mathbf{0}},\left.\quad \mathbf{w}\right|_{\sigma^{*}}=0
$$

Here $\mathbf{w}$ is the vector of the additional displacements and the dot denotes linear increments due to the superposition of additional displacements. Linearization of boundary conditions in $\sigma_{k}$ yields

$$
\left.\mathbf{n} \cdot \mathbf{D}^{*}\right|_{\sigma_{k}}=-\left.p_{k} \cdot J \mathbf{n} \cdot \mathbf{C}^{-T}\right|_{\sigma_{k}}-\left.p_{k} J \mathbf{n} \cdot \mathbf{C}^{-T} \cdot\left(\nabla \cdot \mathbf{w} \mathbf{E}-\nabla_{\mathbf{w}}{ }^{T}\right)\right|_{\sigma_{k}}
$$

In (2.2) the $\nabla$ is the nabla-operator in the deformed state, related to $\nabla^{\circ}$ by the formula $/ 1 /: \nabla=C^{-1} \cdot \nabla^{\circ}$, and $p_{k}{ }^{\circ}$ is the pressure variation in the liquid that is expressed in terms of the additional displacements of the interfacial surface as follows

$$
p_{k}=\frac{d \psi_{k}}{d \rho_{k}} \frac{M_{k}}{V_{k}^{2}} \iint_{\Sigma_{k}} \mathbf{N} \cdot \mathbf{w} d \Sigma=\frac{d \psi_{k}}{d \rho_{k}} \frac{M_{k}}{V_{k}^{2}} \iint_{\sigma_{k}} J \mathbf{n} \cdot \mathbf{C}^{-1} \cdot \mathbf{w} d \mathbf{\sigma}
$$

We note that condition (2.2) differs from the known boundary condition /1/ when the first component, due to the influence of deformation of the elastic solid on the pressure in the liquid, is given for the constant hydrostatic pressure $p_{k}$ on $\sigma_{k}$.

3. As an illustration, we consider the stability of a centrally symmetric deformation of a thick-walled closed spherical shell from a semilinear material filled with a gas and loaded by an external hydrostatic pressure $q$.

Let $r, \varphi, \theta(0 \leqslant \varphi \leqslant 2 \pi,-\pi / 2 \leqslant \theta \leqslant \pi / 2)$ be spherical Lagrange coordinates, and $\mathbf{e}_{r}$, $\mathbf{e}_{\varphi}, \mathbf{e}_{\theta}$ an orthonormal vector basis associated with these coordinates. The centrally symmetric deformation is given in the form

$$
\mathbf{R}=R(r) \mathbf{e}_{r}
$$

From (3.1) we find the gradient of the strain $\mathbf{C}$, the measure of the distortion $\mathbf{U}=\left(\mathbf{C} \cdot \mathbf{C}^{T}\right)^{1 / 3}$, and the rotation tensor $\mathbf{A}=\mathbf{U}^{-1} \cdot \mathbf{C}$

$$
\mathbf{C}=\frac{d R}{d r} \mathbf{e}_{r} \mathbf{e}_{r}+\frac{R}{r}\left(\mathbf{e}_{\varphi} \mathbf{e}_{\varphi}+\mathbf{e}_{\theta} \mathbf{e}_{\theta}\right), \mathbf{U}=\mathbf{C}, \mathbf{A}=\mathbf{E}
$$

Satisfaction of the condition $\operatorname{det} \mathrm{C}>0$ is required here from $R(r)$.

The governing relationship of the medium filling the cavity is taken in the form of the equation of state of an ideal gas in an isothermal process (since there is one cavity we omit 
the subscript $k$ )

$$
p=\psi(\rho) \equiv p_{0} / \rho_{0} \rho
$$

Here $\rho_{0}$ and $p_{0}$ are the gas density and the pressure therein in the reference configuration, which are considered known. We note that the reference configuration in which the elastic body occupies the volume $v$ is not stress-free.

The constitutive law of a semilinear material is written as follows /1/:

$$
\mathbf{D}=\left(\lambda\left(\operatorname{tr} \mathbf{U}^{\prime}-3\right)-2 \mu\right) \mathbf{A}^{\prime}+2 \mu \mathbf{C}^{\prime}
$$

where $\lambda$ and $\mu$ are elastic constants. The strain gradient $\mathbf{C}^{\prime}$, the measure of the distortion $\mathbf{U}^{\prime}$, and the rotation tensor $\mathbf{A}^{\prime}$ in (3.4) are measured from the stress-free state of the elastic body $v^{\prime}$.

We examine the reference configuration $v^{\prime}$ that agrees with this reference configuration $v$ for $p_{0}=0$. The configuration $v$ can be obtained from $v^{\prime}$ by centrally-symmetric deformation under the effect of the constant hydrostatic pressure $p_{0}$ given on the surface of the cavity and the insertion of a gas in this cavity under the pressure $p_{0}$. Let $r^{\prime}, \varphi^{\prime}, \theta^{\prime}$ be spherical coordinates in $v^{\prime}$. We write the mentioned deformation in a form analogous to $(3.1): \quad r=$ $R_{1}\left(r^{\prime}\right) \mathbf{e}_{r}$. We likewise introduce the deformation $\mathbf{R}=R_{2}\left(r^{\prime}\right) \mathbf{e}_{r}$ from $v^{\prime}$ in the desired deformed configuration $V$.

Then the function $R(r)$ in (3.1) can be determined in terms of the functions $R_{1}$ and $K_{2}$ as follows: $R(r)=R_{2}\left(R_{1}^{-1}(r)\right)\left(R_{1}^{-1}\right.$ is the inverse function to $\left.R_{1}\right)$. A governing relationship in the form of (3.4) can be used to find $R_{1}$ and $R_{2}$. The functions $R_{1}$ and $R_{2}$ are known solutions of the Lamé problem for a hollow sphere $/ 1,2 /$

$$
R_{\alpha}=c_{\alpha} r^{\prime}+d_{\alpha} / r^{\prime 2}, \alpha=1,2
$$

The boundary conditions to determine the constants $c_{\alpha}, d_{\alpha}(\alpha=1,2)$ have the form

$$
\begin{aligned}
& (3 \lambda+2 \mu)\left(c_{1}-1\right)-4 \mu d_{1} / b^{3}=0 \\
& (3 \lambda+2 \mu)\left(c_{1}-1\right)-4 \mu d_{1} / a^{3}=-p_{0}\left(c_{1}+d_{1} / a^{3}\right)^{2} \\
& (3 \lambda+2 \mu)\left(c_{2}-1\right)-4 \mu d_{2} / b^{3}=-q\left(c_{2}+d_{2} / b^{3}\right)^{2} \\
& (3 \lambda+2 \mu)\left(c_{2}-1\right)-4 \mu d_{2} / a^{3}=-p\left(c_{2}+d_{2} / a^{3}\right)^{2}
\end{aligned}
$$

Here $a$ is the cavity radius, and $b$ is the radius of the exterior shell surface in the configuration $v^{\prime}$.

Relationship (1.5) to determine the pressure $p$ is and ideal gas in the case of central symmetry takes the form

$$
p=p_{0} R_{1}{ }^{3}(a) / R_{2}{ }^{3}(a)
$$

We note that the system of Eqs.(3.6) to determine the constants $c_{1}$ and $d_{1}$ has no solutions for $p_{0}>p_{0}^{*}$, where

$$
p_{0}^{*}=2 \mu\left(1-x^{3}\right)\left(2+4 x^{3} \frac{1-2 v}{1+v}\right)^{-1}, \quad x=\frac{a}{b}, \quad v=\frac{\lambda}{2(\lambda+\mu)}
$$

A solution exists for $p_{0} \leqslant p_{0}$ * but is not determined in a unique manner. The dependence $R_{1}(a) / a$ on $\bar{p}_{0}=p_{0} /(2 \mu)$ is given in Fig.l for $\nu=0.3$ and different $x$. The dashes denote those parts of the curves where the condition $\operatorname{det} \nabla^{\circ} \mathbf{R}_{1}>0$ is violated. The values $x=0.7 ; 0.95$ correspond to curves 1,2 . It can be verified that the mentioned violation of the uniqueness of the solutionis not associated with violation of the Hadamard condition $/ 1 /$.

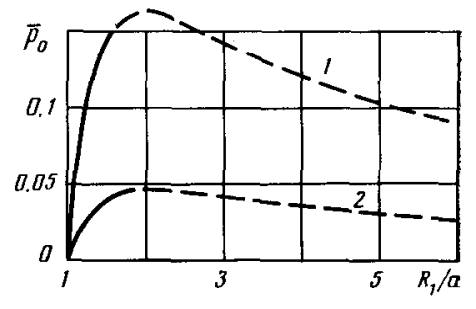

F1g. 1
To analyse buckling, we write the neutral equilibrium in the case of a semilinear material

$\nabla^{\circ} \cdot \mathbf{D}^{*}=\lambda \nabla^{\circ} \nabla^{\circ} \cdot \mathbf{w}+2 \mu \nabla^{\circ} \cdot \nabla^{\circ} \mathbf{v}+(\lambda(\operatorname{tr} \mathbf{U}-3)-2 \mu) \nabla^{\circ} \cdot \mathbf{A}^{\bullet}$

Following $/ 2 /$, we obtain their solution in the form ( $P_{n}$ is a Legendre polynomial)

$$
\begin{gathered}
\mathrm{w}=U_{n}(r) P_{n}(\sin \theta) \mathbf{e}_{r}+W_{n}(r) P_{n}^{\prime}(\sin \theta) \cos \theta \mathrm{e}_{\theta} \\
U_{n}(r)=n r^{n-1} \Phi_{n}(r)-(n+1) r^{-n-2} F_{n}(r) \\
W_{n}(r)=r^{n-1} \Phi_{n}(r)-r^{-n-2} F_{n}(r), \Phi_{n}(r)= \\
\frac{1}{2 n+1} \int_{a}^{r}\left((n+1) \mathrm{X}_{n}(r)+\varphi_{n}(r)\right) r^{-n+1} d r+C_{n} \\
F_{n}(r)=\frac{1}{2 n+1} \int_{a}^{r}\left(n \mathrm{X}_{n}(r)-\varphi_{n}(r)\right) r^{n+2} d r+D_{n}
\end{gathered}
$$




$$
\begin{aligned}
& p_{n}(r)-(n+1) A_{n} r^{n}+\frac{n B_{n}}{r^{n+1}}, \quad \mathrm{X}_{n z}(r) \cdots= \\
& \frac{\lambda-2 \mu}{g(r)}\left(A_{n} r^{n}+\frac{B_{n}}{r^{n+1}}\right), g(r)=2 \mu+\frac{\lambda \operatorname{tr} U-3 \lambda-2 \mu}{R_{2}{ }^{2}+R_{2 j} r}
\end{aligned}
$$

$n=1,2, \ldots$

where $A_{n}, B_{n}, C_{n}, D_{n}$ are arbitrary constants.

In the example under consideration the quantity $p$ defined by (2.3) equals zero. This follows from the form of the subcritical state (3.1), the form (3.8) of the perturbed solution and the orthogonality of the Legendre polynomials. The boundary conditions are the following system of relationships:

$$
\begin{aligned}
& (2 \mu-\beta) U_{n}^{\prime}(b)+(\lambda+\beta) \varphi_{n}(b)=0 \\
& g(b) \mathrm{X}_{n}(b)+b^{-1}(2 \mu-\beta)\left(U_{n}(b)-W_{n}(b)\right)=0 \\
& (2 a-\alpha) U_{n}^{\prime}(a)+(\lambda+\alpha) \varphi_{n}(a)=0 \\
& g(a) \mathrm{X}_{n}(a)+a^{-1}(2 \mu-\alpha)\left(U_{n}(a)-W_{n}(a)\right)=0 \\
& \left(\alpha=p R_{2}(a) / a, \beta=q R_{2}(b) / b\right)
\end{aligned}
$$

The bifurcation pressure $q^{*}$ is found from the condition of non-trivial solvability of this system of linear algebraic equations for the constants of integration' $A_{n}, B_{n}, C_{n}, D_{n}$. The value of $v$ was set equal to 0.3 for the numerical computations.

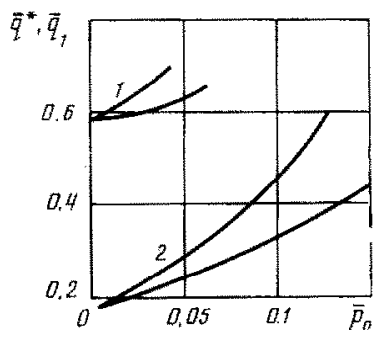

Fig. 2

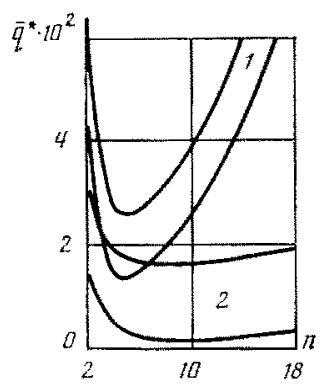

Fig. 3

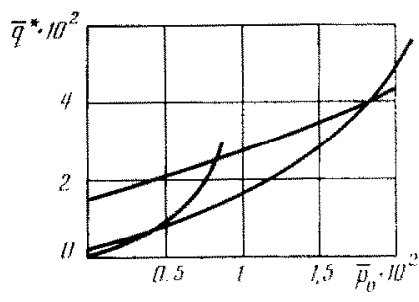

Fig. 4

In order to clarify the influence of the pressure change in the liquid during deformation on the value of the bifurcation pressure $q^{*}$, the computations were also performed in the case when a constant hydrostatic pressure equal to the pressure $p_{0}$ in the liquid in the reference configuration $v$ on the inner surface is given. This corresponds to neglecting the influence of pressure changes in the 1iquid on $q^{*}$. The corresponding bifurcation pressure is denoted by $q_{1}{ }^{*}$. The results of computations showed that $q_{1}^{*} \leqslant q^{*}$. Fig.2 shows the dependence of $q_{1}^{*}$ and $q^{*}$ on $p_{0}$ for $x=0.4$ (curve 1 ) and $x=0.7$ (curves 2). Here and henceforth, the dimensionless pressures, i.e., the pressures referred to $2 \mu$, are marked with a bar.

The $q_{1}^{*}$ and $q^{*}$ above were understood to be the least values in $n$. The numbers $n$ corresponding to these pressures increase as $x$ grows and, as a rule, agree and are independent of $p_{0}$. The dependence on $p_{0}$ appeared in the case $x=0,7$. Precisely for $\ddot{p}_{0}>0.13$ the numbers corresponding to the minimal pressure in $n$ was replaced by $n=2$ from $n=3$. The same replacement occurs for $\tilde{p}_{0}>0.16$ for the bifurcation pressure $q_{1}^{*}$. The remaining values of $n$ as a function of $x$ are presented below

$$
\begin{array}{lllll}
x=0.4 & 0.7 & 0.91 & 0,97 & 0.99 \\
n=2 & 3 ; 2 & 5 & 10 & 18
\end{array}
$$

A graph of the dependence of $q^{*}$ on $n$ is represented in Fig. 3 . Here the function of the discrete argument $n$ is represented by a continuous curve for clarity. As po increases the curves of the dependence of $q^{*}$ on $n$ are shifted upward, while qualitatively unchanged. Curves 1 correspoind to $x=0.91, \bar{p}_{0}=0 ; 0.01$ and curves 2 to $x=0.97$ and $\bar{p}_{0}=0 ; 0.01$.

Graph of the dependence of $q^{*}$ on $\tilde{p}_{\mathfrak{s}}$ are presented in Fig.4 for different values of $x$ The shallower curves corxespond to smaller values of $x$ which are taken equal to $0.91,0.97$, 0.99. The presence of intersections in Fig.4 means that for sufficiently large $\tilde{p}_{0}$ the bifurcation pressures $q^{*}$ can agree for shelis of different thickness.

\section{REFERENCES}

1. LUR'E A.I., Non-linear Theory of Elasticity. Nauka, Moscow, 1980.

2. LUR'E A.I., Theory of Elasticity. Nauka, Moscow, 1970. 